\title{
CAUSAS SOCIALES DE LA DEPRESIÓN Una revisión crítica del modelo atributivo de la depresión
}

\author{
SOCIAL CAUSES OF DEPRESSION \\ Reviewing the Attribution Model of Depression
}

\author{
José Luis Álvaro Estramiana \\ Universidad Complutense de Madrid. España. \\ jlalvaro@cps.ucm.es
}

\author{
Alicia Garrido Luque \\ Universidad Complutense de Madrid. España \\ algarrid@cps.ucm.es
}

\author{
InGe SCHWEIGER Gallo \\ Universidad Complutense de Madrid. España \\ ingesg@cps.ucm.es
}

\begin{abstract}
ResUmen
La depresión es la principal causa de deterioro en el bienestar psicológico en el mundo. La presente investigación tuvo como objetivo abordar el estudio de la depresión desde una perspectiva psicosociológica, analizando el papel que desempeñan las atribuciones causales en su génesis. Para ello, se propone un modelo explicativo de la depresión que tiene en cuenta el papel de factores de naturaleza social, como los acontecimientos vitales estresantes y los recursos de afrontamiento, el apoyo social, la alienación y la autoestima. Los resultados obtenidos son consistentes con la hipótesis de un estilo atributivo característico, así como con el modelo atributivo de la depresión. Sin embargo, también apuntan a que el estilo atributivo no está significativamente asociado a la depresión cuando tenemos en cuenta el papel de otras variables sociales. Estos resultados indican la necesidad de una perspectiva más amplia, y específicamente sociológica, en los estudios sobre la depresión.
\end{abstract}

\section{Palabras Clave}

Alienación, Atribución causal, Autoestima, Bienestar psicológico, Estrés social.

\section{Abstract}

Depression is the leading cause of deterioration in psychological well-being in the world. The present research aimed at analyzing depression from a socio-psychological perspective, focusing on the role of causal attributions on the origins of depressive disorders. Therefore, a model of depression is suggested in which the role of social factors such as stressful life events and coping strategies, social support, alienation and self-esteem 
are considered. The results are consistent with the attributional style hypothesis, as well as with the attribution model of depression. However, the results also point to the fact that the attributional style is not significantly associated to depression when taking into consideration the role of other social variables. These results lead us to the conclusion that a larger, and specifically more sociological, perspective is needed when analyzing depression.

\section{KEYWORDS}

Alienation, Causal Attribution, Psychological Well-being, Self-esteem, Social Stress.

\section{INTRODUCCIÓN}

La depresión, tal y como nos dice la Organización Mundial de la Salud (OMS), es la principal causa de deterioro en la salud mental y afecta a 121 millones de personas en el mundo (World Health Organization 2007). Concretamente, un reciente estudio europeo muestra que la depresión y las fobias específicas constituyen los problemas mentales más comunes en el continente europeo, con una prevalencia del 13\% (Alonso et al. 2004).

Existen distintas formas de entender la depresión. Desde un enfoque clínico, puede ser definida como un trastorno de la personalidad de carácter afectivo, cognitivo y comportamental. Desde un punto de vista conductual, se entiende la depresión como la consecuencia de la falta de refuerzo o de la no contingencia entre conducta y refuerzo, mientras que la teoría cognitiva concibe la depresión como resultado de pensamientos inadecuados, distorsionando el individuo la realidad de forma negativa. Los criterios de diagnóstico varían del mismo modo, aunque generalmente incluyen la presencia de síntomas, la duración de los mismos y una actividad disfuncional en diversas áreas sociales como el trabajo, las relaciones interpersonales, familiares, etc.

En este artículo trataremos la depresión como un problema psicológico y social, caracterizado por un sentimiento de malestar afectivo y físico. Se trata de un problema psicológico, porque es la persona quien la sufre, y social porque no es sino en el contexto social del individuo donde dicha experiencia cobra sentido. Como estado afectivo negativo vendría caracterizado por manifestaciones de tristeza, desmoralización, aislamiento, desesperanza, fracaso y pensamientos de suicidio, mientras que el malestar físico haría referencia a problemas como la falta de apetito, la pérdida de peso, las dificultades para conciliar el sueño, el agotamiento, etcétera.

Por tanto, la depresión, tal y como aquí la entendemos, no se refiere a un trastorno de la personalidad, sino a un estado subjetivo de malestar físico y emocional, entendido como el resultado de las presiones socioestructurales sobre el individuo (Mirowsky y Ross 1989). Por ello, estamos interesados, más que en establecer categorías excluyentes sobre la base de un criterio rígido de diagnóstico de la depresión, en conocer las causas de la variabilidad en síntomas asociados a la misma, independientemente de que justifiquen o no un criterio de diferenciación. De esta forma, es posible indagar acerca de las causas de dicho deterioro y sólo así tiene sentido preguntarse por los factores que explican por qué unas personas o grupos sociales tienen una mayor probabili- 
dad de sufrir mayores síntomas depresivos que otras y por qué los síntomas asociados a la depresión no se distribuyen de forma aleatoria entre la población.

\section{PROCESOS COGNITIVOS, ATRIBUCIONES Y DEPRESIÓN}

Hasta la década de 1970, los principales enfoques psicológicos explicativos de la depresión o bien procedían del psicoanálisis o estaban basados en las teorías del aprendizaje. La crisis del conductismo y su paulatina sustitución por el paradigma cognitivista influirá en los modelos teóricos que tratan de dar cuenta de la aparición de la depresión. Estos comienzan, a partir de finales de 1960, no sólo a reconocer sino también a enfatizar el papel que algunos factores de naturaleza mental o cognitiva juegan en la génesis de la depresión. Dentro del ámbito de la psicoterapia, uno de los enfoques más influyentes fue el de Beck (1967; 1976), basado en el supuesto de que existe una relación causal unidireccional entre el sistema de creencias de la persona y sus afectos y emociones. Según este autor, la base de la depresión se encuentra en un triple déficit en el sistema de creencias que lleva a la persona a percibirse a sí misma, al mundo y al futuro en términos negativos. Esta tríada cognitiva, que tiene su origen en las experiencias tempranas del individuo, implica errores sistemáticos en el pensamiento y distorsiones en el procesamiento de la información. Según Beck (1967; 1976), existe un determinado grado de vulnerabilidad o tendencia a la depresión, que es el resultado de la activación de determinados esquemas, constituidos por actitudes disfuncionales y pensamientos automáticos que desembocan en una percepción distorsionada de la realidad.

Situándonos en el ámbito de la psicología social, el giro cognitivista que se produjo en la psicología también tuvo un reflejo en las explicaciones de la depresión, que comienzan a buscar referentes teóricos en las teorías de la atribución (Álvaro y Páez 1996; Garrido y Álvaro 2007). Hasta finales de la década de 1970, uno de los modelos más utilizados para explicar la depresión fue la teoría de la indefensión aprendida de Seligman (1975), que se situaba, si bien de forma crítica, dentro de las coordenadas del condicionamiento operante skinneriano. En este modelo, la depresión es el resultado de la exposición de la persona a situaciones en las que no existe un control sobre las consecuencias del comportamiento. Esta percepción de incontrolabilidad provocará una disminución de las respuestas, interferirá con la adquisición de respuestas nuevas y determinará la aparición de sentimientos depresivos. Seligman utiliza el término de indefensión aprendida para denominar al estado psicológico caracterizado por la presencia de este triple déficit motivacional, cognitivo y emocional y establece un paralelismo entre dicho estado y la depresión reactiva. La teoría de la indefensión aprendida subraya, por tanto, la importancia que tienen para la salud mental los sentimientos de control sobre el medio.

Los planteamientos iniciales de Seligman (1981) dieron paso a sucesivas reformulaciones en las que se percibe claramente la influencia del giro cognitivista en la psicología social. Una de las primeras fue la propuesta por Abramson, Seligman y Teasdale (1978), 
en la que el análisis de la indefensión se sitúa dentro de los parámetros de las teorías de la atribución. A la hora de analizar las bases de la depresión no sólo se tiene en cuenta la dimensión interno/externo, sino otras dos dimensiones, como la estabilidad/inestabilidad o la especificidad/globalidad; un planteamiento que se encuentra en consonancia con la teoría de la atribución de Weiner (1986). Mediante la utilización de la dimensión estabilidad/inestabilidad puede establecerse una distinción entre aquellos casos en los que los déficit asociados al estado de indefensión tienen un carácter crónico (asociado a causas estables) y aquellos otros en los que la inestabilidad de las causas lleva a un estado de indefensión transitorio. Una tercera dimensión hace referencia a la globalidad o especificidad de los factores utilizados por la persona para explicar su situación. Dependiendo de que las causas sean globales o específicas, los déficit propios del estado de indefensión se transferirán a otras situaciones o quedarán circunscritos a una situación determinada. De acuerdo con estos autores, las personas que tienden a explicar acontecimientos negativos atribuyéndolos a causas internas, estables y globales tienen una mayor probabilidad de mostrar déficit depresivos. Esta primera reformulación no elimina el papel de la indefensión en la aparición de la depresión, pero sí reduce su importancia.

En la investigación posterior y en el propio trabajo de Seligman (1979) los procesos de atribución comienzan a ser considerados independientemente de la condición de indefensión y surge el estilo atributivo depresivo (atribuciones internas, estables y globales para sucesos negativos y atribuciones externas, inestables y específicas para sucesos positivos) como factor causal fundamental. La consecuencia final, tal y como reconoce el propio Seligman (Peterson, Maier y Seligman 1993), es el cambio de interés del ambiente incontrolable a los sucesos negativos y la explicación que de ellos construyen las personas. El giro cognitivo a la teoría de la indefensión aprendida se completa con una segunda reformulación en la que se señala a la desesperanza como causa suficiente de los síntomas de la depresión (Abramson, Alloy y Metalsky 1988; 1995). Por desesperanza se entiende la expectativa de que resultados altamente deseados no sucederán y/o resultados altamente negativos tendrán lugar, no existiendo respuesta en el propio repertorio de conductas que pueda cambiar la probabilidad de que estos sucesos ocurran.

En resumen, el modelo atributivo de la depresión se ha convertido en uno de los modelos más utilizados para explicar la sintomatología depresiva, argumentando que las personas que realizan atribuciones internas, estables y globales sobre acontecimientos negativos de su vida cotidiana tienen una mayor probabilidad de sufrir una depresión. Frente a este modelo característico de una psicología social de corte psicológico, existen otras perspectivas más sociológicas en las que la depresión es vista como la respuesta del individuo a las condiciones del medio, relacionando la depresión con la posición que la persona ocupa en la estructura social y con sus propias interacciones sociales (Aneshensel y Phelan 1999; Blanco y Díaz 2007; Ross y Sastry 1999; Sánchez, Garrido y Álvaro 2003). 


\section{Causas sociales de LA Depresión}

En general, los modelos teóricos que enfatizan el papel de los procesos cognitivos en la génesis de la depresión parten de una concepción de la persona como un ser racional, que procesa de forma consciente y voluntaria la información procedente del medio, siendo este procesamiento de la información la base de la construcción de sus creencias. El deterioro psicológico se explicaría por un fallo en el sistema de procesamiento de la información, que genera en las personas depresivas un sistema de creencias prototípico, caracterizado por la inferencia arbitraria y la distorsión de la realidad. Esta forma de entender la etiología del deterioro psicológico ignora los determinantes sociales de la depresión al situar su origen en el interior de la mente de las personas.

Aunque menos numerosos, algunos modelos teóricos e investigaciones han destacado el papel de factores sociales en la depresión (Álvaro, Torregrosa y Garrido 1992; Blanco y Díaz 2007, Brown y Harris 1978; Cochrane 1983; McLeod y Nonnemaker 1999; Sánchez, Garrido y Álvaro 2003; Yu y Williams 1999). Uno de los objetivos de este estudio es mostrar cómo la no inclusión de factores sociales en el origen de la depresión ha llevado a una errónea consideración del papel de los procesos de atribución en la causación de los déficit depresivos. Aquí consideraremos ciertos factores como los estresores y las estrategias de adaptación, el apoyo social, la alienación y la autoestima como indicadores de la posición social de las personas y, por tanto, determinantes de la variabilidad observada en los niveles de depresión.

El estrés puede ser definido como el conjunto de demandas sociales y presiones del medio que sobrepasan la capacidad del individuo para ajustarse a las mismas. Dichos constreñimientos estructurales limitan la capacidad de acción y suponen una amenaza al equilibrio psicológico (Wheaton 1999). Son numerosos los estudios que ponen de manifiesto la relación entre los acontecimientos vitales estresantes y la depresión (Gotlib y Hammen 1992; Pearlin 1999; Sánchez, Álvaro y Garrido 2000; Wheaton 1999). Si bien la noción de acontecimientos estresantes nos remite a situaciones interpersonales o sociales que tienen un impacto negativo en el bienestar psicológico de las personas, los estudios que abordan el impacto de los efectos de acontecimientos negativos sobre el individuo han estado vinculados al análisis de aquellas estrategias que son más relevantes para poder mitigar sus consecuencias negativas. Sin duda, entre los modelos más estudiados se encuentra el modelo transaccional del estrés de Lazarus y Folkman (1986). En este modelo, el efecto de los estresores depende no sólo de la evaluación que el individuo hace del medio, sino también de los recursos de adaptación de los que pueda disponer. En resumen, los estudios sobre el impacto del estrés deben ser estudiados de forma conjunta con las estrategias de afrontamiento de las personas. Dichas estrategias son recursos que hacen que el impacto del estrés no sea el mismo para todo el mundo. Muy vinculado a los acontecimientos vitales estresantes se encuentra el apoyo social. Independien- 
temente de que actúe en presencia de estresores, disminuyendo su impacto, o que tenga un efecto directo sobre la salud, son numerosos los estudios que constatan sus efectos beneficiosos en el bienestar psicológico (por ejemplo, Turner y Turner 1999).

Por otro lado, la inclusión de variables como la alienación y la autoestima permiten ampliar una perspectiva psicosociológica en el estudio de los estados emocionales de las personas. Desde la sociología, la alienación es una categoría analítica básica para entender las condiciones materiales de desigualdad social que la generan -el trabajo alienado en la obra de Marx hace referencia a las condiciones en las que se produce el trabajo productivo en el sistema capitalista (Marx 1980; 1978)-. En él, el trabajador no decide sobre los objetos producidos, ni sobre el proceso de producción y los resultados del mismo, convirtiéndose así en mera mercancía. El concepto de alienación está íntimamente ligado al de falta de control (Ross y Sastry 1999) y ha sido tratado desde el punto de vista del sujeto, desde su propia experiencia vital. Así, según Seeman $(1959 ; 1983)$ pueden diferenciarse cinco dimensiones diferentes de la alienación: indefensión, carencia de significado, aislamiento, autoestrañamiento y anomia. Estas dimensiones implican todas ellas una pérdida del control personal sobre el medio y hacen referencia a los efectos de la deficiente integración del individuo en su sociedad. Este debilitamiento de las relaciones del individuo con el marco moral de la colectividad está vinculado a otro concepto de la tradición sociológica como es el de anomia, desarrollado por Durkheim en obras como La división del trabajo social y El suicidio, publicadas respectivamente en 1893 y 1897.

Un individuo alienado está despojado de su capacidad de influir sobre su medio, ya que actúa en un vacío normativo, simbólico e interpersonal. La falta de expectativas para transformar las circunstancias y sucesos adversos, la incapacidad para predecir el futuro, la no existencia de normas sociales, la ausencia de significado y propósito en la vida personal, así como el sentimiento de separación y distanciamiento con respecto a sus ideas, acciones y sentimientos, sujetos al control o decisión de otros, hunden sus raíces en las condiciones sociales de existencia fijadas por la posición social. Sin duda, los elementos anteriormente descritos coinciden, en gran medida, con algunas de las características del modelo de bienestar social de Keyes (1998), para quien la integración social, la contribución social, entendida como el sentimiento de que se puede contribuir de forma valiosa a la sociedad, la aceptación social, la evaluación de las instituciones sociales como marco propicio para el desarrollo personal (actualización social) y el interés y preocupación por dar un sentido al entorno social (coherencia social) constituyen los factores esenciales del bienestar social.

Asimismo, la autoestima, como valoración de la identidad personal, depende de las actitudes de los otros significativos: la autoimagen no se da en un vacío social, sino que es a través de la interacción con otros como tomamos conciencia de nosotros mismos. La identidad del yo y la autoimagen se dan en el proceso de interacción simbólica entre las personas, el cual nos remite a las posiciones sociales de los sujetos que interactúan y a la estructura social de la que forman parte (Stryker 1980). La autoimagen está condicionada por los procesos de socialización diferenciales que se 
dan en las distintas clases sociales. Tal y como ponen de manifiesto autores como Rosenberg (1965), la autoestima viene determinada por la clase social a la que pertenecemos. Psicólogos sociales como Cochrane (1983) han puesto de manifiesto que tanto las posibilidades de tener que enfrentarse a situaciones de estrés, como los recursos de afrontamiento ante las mismas, entre los que se encuentra la autoestima, varían según las clases sociales. La falta de control personal está asociada a una reacción adaptativa que incluye una actitud de baja autoestima. En este contexto, la depresión se considera como una respuesta más dentro de un repertorio de posibles respuestas. Ahora bien, el desarrollo de síntomas es una forma de afrontamiento típica de determinados grupos sociales, precisamente aquellos que ocupan posiciones inferiores en la estructura social. La respuesta más efectiva, funcional y socialmente deseable está constituida por mecanismos de defensa como una alta autoestima, respuesta típica de grupos sociales favorecidos (aquellos que ocupan un alto estatus social). Las personas pertenecientes a estos grupos sociales mostrarán una menor vulnerabilidad, resultado de la disponibilidad de recursos de afrontamiento efectivos.

En resumen, mientras las teorías cognitivas han ido enfatizando la importancia de la representación que las personas elaboran del mundo, los modelos de causación social, a través de conceptos como los de estrés social y alienación, destacan el papel de nuestras relaciones normativas, simbólicas y materiales con el ambiente. Al mismo tiempo que la investigación cognitiva tiende a considerar la existencia de una baja autoestima como síntoma de la depresión, como si se tratara básicamente de un sesgo cognitivo relacionado con la percepción del yo, los modelos sociológicos la sitúan como un proceso mediador entre la estructura social y la depresión; una variable que se construye en las relaciones sociales y que tiene un efecto sobre el bienestar psicológico del individuo. Las experiencias de estrés, junto con los factores de amortiguación y afrontamiento, entre los que debemos incluir el apoyo social, en conjunción con la alienación y la autoestima, dependen de la posición social, de las condiciones materiales de existencia que exponen a las personas a situaciones de dominio o de falta de control sobre el medio. Es esta falta de control el mecanismo a través del cual podemos explicar el vínculo entre estresores, alienación, autoestima y depresión.

El objetivo de este artículo es analizar y revisar el papel que desempeñan las atribuciones causales en la génesis de los trastornos depresivos. Más específicamente, se pretende realizar un análisis crítico del modelo atributivo de la depresión que señala que la atribución de causas internas, estables y globales ante circunstancias adversas o acontecimientos vitales estresantes está asociada a la depresión. En dicha crítica se señala la insuficiencia de dicho modelo cognitivo por no haber tenido en cuenta un análisis de las variables de carácter sociológico antes señaladas. Todo modelo explicativo de la depresión debe tener en cuenta el papel de factores de naturaleza social, como son el estrés y las estrategias de adaptación al mismo, entre las que incluimos el apoyo social, así como los sentimientos de alienación y autoestima. 


\section{MÉTOdo}

\section{Participantes}

Participaron 401 sujetos de diferentes edades, sexo, ingresos, nivel profesional y nivel educativo. Un $56.6 \%$ eran varones y un $44.4 \%$ mujeres. Del total de la muestra, el $53.1 \%$ de los participantes estaba trabajando, un $12.7 \%$ se encontraba en paro, un $12.5 \%$ estaban jubilados, el $13.7 \%$ eran estudiantes y un $7.7 \%$ eran amas de casa. En cuanto al nivel educativo, el $22.7 \%$ no había finalizado el graduado escolar, el $38.2 \%$ había finalizado sus estudios de bachillerato o de formación profesional y el $38.2 \%$ tenía estudios universitarios. Los ingresos económicos oscilaban entre menos de 600 euros y más de 1.500 euros.

\section{Instrumentos}

Para la obtención de los datos se elaboró un cuestionario autoadministrado. Nuestra intención fue obtener una amplia variabilidad muestral en la variable dependiente de la depresión, por lo que se optó por un muestreo no probabilístico con el objetivo de seleccionar una muestra heterogénea en la que estuviesen representadas todas las clases sociales, grupos de edad y sexo. Además de las variables sociodemográficas ya comentadas, se incluyeron medidas de alienación, acontecimientos estresantes, estrategias de afrontamiento y apoyo social, autoestima, estilos de atribución y depresión.

Para la operacionalización de la variable depresión se utilizó el cuestionario de depresión de Beck (1967; Beck, Rush, Shaw y Emery 1983) que consta de 21 ítems. En la muestra de este estudio se obtuvo un coeficiente alpha de consistencia interna de 0.89. Para la medición de los estresores se utilizó el cuestionario de 32 ítems de Herrero, Gracia y Musitu (1996), que mostró una consistencia interna (Cronbach 1951) de 0.85. Para las estrategias de afrontamiento el cuestionario utilizado fue el de Folkman y Lazarus (1980), para el que se obtuvo un alfa de Cronbach de 0.92. Este cuestionario se subdivide en ocho escalas que reflejan ocho formas de enfrentamiento a las situaciones de estrés (confrontación, reevaluación positiva, autocontrol, planificación, búsqueda de apoyo social, aceptación de la responsabilidad, distanciamiento y huida). La medición del apoyo social se realizó mediante una adaptación del cuestionario de Herrero, Gracia y Musitu (1996) para el que se obtuvo un alfa de consistencia interna de 0.85 (Cronbach 1951). Para la evaluación de la alienación se adaptó el cuestionario de alienación de Khon y Schooler (Khon 1976). Dicho cuestionario consta de 16 ítems y su diseño se deriva del trabajo de Seeman (1959; Seeman 1983). El coeficiente alpha de consistencia interna para esta escala fue 0.85 . En cuanto a la autoestima, se utilizó una escala de 8 ítems (Warr y Jackson 1983), cuyo coeficiente alpha de consistencia interna fue de 0.77. Para la operacionalización de los estilos de atribución se empleó el cuestionario de estilo de atribución (Peterson et al. 1982). El coeficiente alpha de consistencia interna obtenido fue de 0.87 . 


\section{Procedimiento}

Los cuestionarios fueron autoadministrados, salvo a determinados grupos, en función de sus características sociodemográficas, como los mayores de 60 años o las personas con un bajo nivel educativo. La duración media para contestar a los mismos fue de unos 45 minutos. A los entrevistados se les informó del carácter anónimo de las respuestas, así como de los objetivos de las mismas.

\section{Resultados}

En primer lugar, los resultados obtenidos son inicialmente consistentes con la hipótesis de un estilo atributivo característico. Las personas que tienden a atribuir las causas de sucesos negativos a factores internos tenderán también a generalizar dichas causas a otras áreas de la vida $(r=0.50, p<0.001)$, así como a percibir dichas causas como duraderas en el tiempo $(r=0.46, p<0.001)$. En segundo lugar, los resultados son congruentes con el modelo atributivo de la depresión. La depresión muestra una correlación positiva con la internalidad $(r=0.32, p<0.001)$, la estabilidad $(r=0.38, p<0.001)$ y la globalidad $(r=0.40, p<0.001)$ de las causas que se utilizan para explicar sucesos negativos. Los tamaños de los efectos de las correlaciones entre las variables estabilidad y depresión $(d=0.82)$ y globalidad y depresión $(d=0.88)$ son grandes, mientras que en el caso de la correlación entre internalidad y depresión el tamaño del efecto $(d=0.67)$ puede calificarse de medio (Cohen 1988). Al igual que en otras investigaciones, las correlaciones son significativas, situándose por encima de las correlaciones medias (0.30) observadas en otros estudios (Sweeny, Anderson y Bayley 1986). En tercer lugar, observamos que la variabilidad de las puntuaciones en depresión, recogida por los factores cognitivos de atribución es, sin embargo, limitada. En este estudio, la varianza explicada por los factores cognitivos es del $26 \%$.

Finalmente, los análisis de regresión múltiple realizados muestran que el estilo atributivo no está significativamente asociado a la depresión. Los coeficientes beta estandarizados $(\beta)$ indican que las variables que resultaron predictores significativos de la depresión son los acontecimientos vitales estresantes $(\beta=0.23, p<0.01)$, la alienación $(\beta=0.21, p<0.01)$, la autoestima $(\beta=-0.29, p<0.01)$ y el apoyo social $(\beta=-0.17$, $p<0.01$ ). Asimismo, entre los estilos de afrontamiento, sólo resultaron significativos los relativos al distanciamiento $(\beta=-0.09, p<0.005)$ y a la huida $(\beta=0.16, p<0.001)$. Por tanto, la inclusión de variables tales como los acontecimientos vitales estresantes y las estrategias de afrontamiento y apoyo social, junto con la alienación y la autoestima, en un modelo explicativo de la depresión, anula el efecto de los estilos atributivos, lo que pone en cuestión los resultados obtenidos en estudios previos sobre el carácter predictivo de las explicaciones causales en el deterioro psicológico característico de la depresión. La varianza explicada por los factores incluidos en el análisis de regresión, considerando también las variables de apoyo social y acontecimientos vitales estresantes es cercana al $60 \%$. 


\section{Discusión}

Tal y como hemos podido constatar en el presente estudio, los resultados obtenidos de carácter correlacional confirman, inicialmente, la hipótesis de un estilo atributivo característico. Entre las personas entrevistadas, aquéllas que atribuyen las causas de sucesos negativos a factores internos (factor de internalidad) también las perciben como duraderas en el tiempo (factor de estabilidad) y generalizan dichas causas a otras áreas vitales (factor de globalidad). Es decir, creer que las causas de un acontecimiento negativo están en uno mismo hace que la persona tenga una alta probabilidad de pensar que dichas causas permanecerán en el tiempo y que son generalizables a otras situaciones 0 ámbitos negativos de la propia existencia.

Asimismo, y de acuerdo con el modelo atributivo de la depresión, ésta se encuentra asociada positivamente tanto a los factores de internalidad, como de estabilidad y globalidad. Es decir, aquellos sujetos que tienen un estilo atributivo caracterizado por los factores anteriormente señalados tendrán una mayor probabilidad de sufrir déficit depresivos. Esta asociación entre estilos atributivos internos, estables y globales y la depresión, observada también en otros estudios (Fresco, Alloy, Reilly-Harrington 2006; Sweeny, Anderson y Bailey 1986) podría tener importantes implicaciones para la prevención y terapia de estados depresivos. Si la depresión está causada por un procesamiento de información característico, el cambio en las atribuciones llevaría a la prevención o terapia de un estado depresivo.

Sin embargo, nuestros resultados indican que la asociación entre el estilo atributivo y la depresión deja de ser significativa una vez controlado el efecto de variables como los acontecimientos estresantes, la autoestima y la alienación -factores estos fuertemente asociados a la posición social- (Sánchez, Garrido y Álvaro 2003). En definitiva, podemos afirmar que cuando las condiciones en las que se desarrolla la vida de las personas están caracterizadas tanto por la presencia de sucesos negativos, como por la alienación, entre cuyas dimensiones podemos incluir el sentimiento de indefensión, la falta de sentido en la vida, la anomia o la ausencia de normas sociales y el sentimiento de extrañamiento cultural (Sánchez, Garrido y Álvaro 2003), y una imagen negativa de uno mismo $o$ una valoración negativa del yo, se incrementan significativamente las probabilidades de sufrir unos mayores niveles de depresión. Asimismo, no debemos descartar el papel que juega el apoyo social y ciertas estrategias de afrontamiento. Así, mientras las personas que muestran un mayor apoyo social y un mayor distanciamiento de los problemas manifiestan menos déficit depresivos, aquellas otras que utilizan como estrategia de afrontamiento la huida de los problemas tienen unos niveles mayores de depresión. Como ya se señaló, tanto la imagen negativa de uno mismo, como las situaciones de estrés y los sentimientos de alienación no surgen en un vacío social, sino que tienen su origen en la interacción social y son consecuencia de las experiencias de incontrolabilidad, falta de sentido, anomia y autoestrañamiento que viven las personas en su relación con el medio material y simbólico. Teniendo en cuenta que los análisis de regresión realizados cuestionan el carácter predictivo de las explicaciones causales de 
tipo atributivo en el deterioro psicológico característico de la depresión, futuros estudios deberán poner a prueba el hecho de que el estilo atributivo negativo pueda darse sólo en aquellos individuos con una baja autoestima (Southall y Roberts 2002) 0 ante acontecimientos incontrolables.

En cuanto a las limitaciones de este estudio, debemos señalar que se trata de un estudio correlacional y que, por tanto, son precisos posteriores estudios longitudinales para poder desentrañar las relaciones de causalidad entre los factores sociales de la depresión y la posible relación entre los estados depresivos y una movilidad social descendente que influya, a su vez, sobre factores como el estrés, la alienación y la autoestima. Por otro lado, la evidencia de que existe una asociación entre el estilo atributivo y la depresión tampoco indica la relación causal entre dichos factores. Frente a la hipótesis según la cual un estilo atributivo vulnerable, en presencia de un acontecimiento estresante, desencadena un proceso depresivo, también es posible afirmar que las personas que sufren un deterioro en su salud mental de tipo depresivo tienden a tener un estilo atributivo caracterizado por la internalidad, la globalidad y la estabilidad, sin que dichos factores actúen necesariamente como precipitadores de los déficit depresivos observados.

\section{Conclusiones}

Como conclusión, proponemos una revisión del modelo atributivo de la depresión. De hecho, los estudios en los que se contempla la asociación entre depresión y atribuciones internas, estables y globales de sucesos negativos no tienen en cuenta factores sociales como causas de la depresión. En presencia de dichos factores, los modelos de atribución pueden carecer de valor predictivo. De ahí que la evidencia correlacional que ha servido de apoyo empírico a los teóricos de la atribución esté sesgada al ignorar las causas sociales de la depresión.

En definitiva, podemos deducir del presente estudio que son las desigualdades sociales, a través de las experiencias de estrés y de ciertas estrategias de afrontamiento, entre las que podemos incluir las redes de apoyo social disponibles, y las diferencias en los sentimientos de alienación y autoestima, y no los estilos atributivos per se, los que tienen un peso significativo en la probabilidad de sufrir una mayor sintomatología depresiva. Futuros estudios deberán analizar en qué medida las experiencias de estrés y los sentimientos de alienación, junto con una baja autoestima, tienen un efecto sobre la depresión y en qué medida la depresión incrementa tanto la vulnerabilidad ante sucesos estresantes como los sentimientos de alienación, e influye en el deterioro de la imagen que tenemos de nosotros mismos y en los recursos disponibles para afrontar las circunstancias adversas. De cualquier forma, tanto los acontecimientos estresantes, como la alienación y la autoestima son factores que no se distribuyen de igual manera entre la población. Son las personas que pertenecen a las clases sociales más desfavorecidas las que tienen una mayor probabilidad de sufrir en sus vidas acontecimientos que inciden sobre el control del medio y, en consecuencia, sobre su autoimagen. Asimismo, 
los recursos para enfrentarse a dichas situaciones y las redes sociales de apoyo varían en función de nuestra posición en la estructura social (Ross y Sastry 1999; Sánchez, Garrido y Álvaro 2003).

En resumen, los modelos cognitivos, como el modelo atributivo de la depresión aquí analizado, son insuficientes para dar cuenta de la depresión. Son necesarios enfoques en los que se destaque la importancia de las desigualdades que el sistema social impone sobre los individuos y nos permitan analizar cómo la salud mental refleja, en muchos casos, la respuesta emocional que las personas dan ante dichas desigualdades sociales. Sin una consideración previa de los desequilibrios en la estructura social, de los problemas sociales a los que las personas se enfrentan en su día a día, no es posible dar cuenta de las diferencias en la salud mental de la población. Sin duda, sin la presencia de variables como el estrés social, la alienación y la autoestima, de honda tradición sociológica, no es posible trazar un mapa explicativo del deterioro psicológico de las personas. Algo ya señalado por otros autores como Keyes (1998) en su definición de bienestar social, al afirmar que los investigadores en este área deben desarrollar estudios en los que se intente vincular el bienestar psicológico con los determinantes sociales del mismo. Los modelos de la atribución aplicados al deterioro psicológico y, más específicamente, a la depresión, no han considerado las variables estructurales en la configuración de los déficit depresivos. La relevancia de dichos modelos en los estudios sobre la depresión revela la importancia de considerarla no tanto como el resultado de un procesamiento de la información con unas características específicas (estilo atributivo interno, estable y global), sino como la consecuencia de las relaciones entre el individuo y su contexto social. En este sentido, promover la salud mental no es sólo una tarea que resida en la capacidad de las personas para cambiar sus estilos cognitivos, algo que en determinados casos no debe quedar excluido, sino en la transformación tanto de las condiciones sociales que influyen en nuestra integración social como de las interacciones sociales en las que se construye nuestra autoestima. En resumen, el modelo atributivo de la depresión es heredero de un modelo psicológico en el que el ser humano y su salud o enfermedad mental aparecen como el resultado de procesos internos de carácter cognitivo y en el que el medio social queda ausente. Las conclusiones de este estudio revelan que dicho modelo cognitivo de explicación de la depresión no es el más adecuado a la hora de revelar los procesos sociales asociados a la depresión. Esto, obviamente, tiene consecuencias en el plano de la intervención, pues pone el énfasis en las medidas que tienen que ver con el desarrollo de instituciones sociales positivas (Díaz, Blanco, Horcajo y Valle 2007). Se trataría de considerar no sólo el cambio individual, sino la intervención comunitaria como forma de cambio social (Álvaro y Páez 1996). 


\section{REFERENCIAS BiBLIOGRÁFICAS}

Abramson, L. Y., L. B. Alloy y G. I. Metalsky. 1988. "The cognitive diathesis-stress theories of depression. Toward an adequate evaluation of the theories' validities". Pp. 3-30 en Cognitive processes in depression, ed. Alloy, L. B. Nueva York: Guilford.

Abramson, L. Y., L. B. Alloy y G. I. Metalsky. 1995. "Hopelessness depression”. Pp. 113-134 en Explanatory style, eds. Buchanan, G. M. y M. E. P. Seligman. New Jersey: Lawrence Erlbaum.

Abramson, L. Y., M. E. P. Seligman y J. D. Teasdale. 1978. "Learned helplessness in humans". Journal of Abnormal Psychology 87: 49-74.

Alonso, J., M. C. Angermeyer, S. Bernet, R. Bruffaerts, T. S. Brugha, H. Bryson et al. 2004. "Prevalence of mental disorders in Europe: results from the European Study of the Epidemiology of Mental Disorders (ESEMeD) project". Acta Psychiatrica Scandinavica 109 (s420): 21-27.

Álvaro, J. L., J. R. Torregrosa y A. Garrido. 1992. "Estructura social y salud mental". Pp. 9-30 en Influencias sociales y psicológicas en la salud mental, eds. Álvaro, J. L., J. R. Torregrosa y A. Garrido. Madrid: Siglo XXI.

Álvaro, J. L. y D. Páez. 1996. "Psicología social de la salud mental". Pp. 381-407 en Psicología social aplicada. Madrid: McGraw-Hill.

Aneshensel, C. S. y J. C. Phelan, eds. 1999. Handbook of the sociology of mental health. Nueva York: Kluwer Academia.

Beck, A. T. 1967. Depression: Clinical, experimental, and theoretical aspects. Nueva York: Harper \& Row.

Beck, A. T. 1976. Cognitive therapy and the emotional disorders. Nueva York: International Universities Press.

Beck, A. T., J. Rush, B. Shaw y G. Emery. 1983. Terapia cognitiva de la depresión. Bilbao: Desclée de Brouwer.

Blanco, A. y D. Díaz. 2007. "Social order and mental health: a social well-being approach" Psychology in Spain 11: 61-71.

Brown, G. W. y T. Harris. 1978. Social origins of depression: A study of psychiatric depression in women. Londres: Tavistock Publications.

Cochrane, R. 1983. The social creation of mental illness. Londres: Longman.

Cohen, J. 1988. Statistical power analysis for the behavioral sciences. Hillsdale, NJ: Erlbaum.

Cronbach, L. J. 1951. "Coefficient alpha and the internal structure of test" Psychometrica 16: 297-334.

Díaz, D., A. Blanco, J. Horcajo y C. Valle. 2007. "Aplicación del modelo del estado completo de salud al estudio de la depresión" Psicothema 19: 286-294. 
Durkheim, E. 1973. De la división social del trabajo. Buenos Aires: Shapire.

Durkheim, E. 1998. El suicidio. Madrid: Akal.

Folkman, S. y R. S. Lazarus. 1980. "An analysis of coping in a middle-aged community simple." Journal of Health and Social Behavior 21: 219-239.

Fresco, D. M., L. B. Alloy y N. Reilly-Harrington. 2006. "Association of attribution style for negative and positive events and the occurrence of life events with depression and anxiety." Journal of Social and Clinical Psychology 25: 1140-1159.

Garrido, A. y J. L. Álvaro. 2007. Psicología social. Perspectivas psicológicas y sociológicas. Madrid: McGraw-Hill.

Gotlib, I. H. y C. L. Hammen. 1992. Psychological aspects of depression: Toward a cognitive-interpersonal integration. Londres: Wiley.

Herrero, J., E. Gracia y G. Musitu. 1996. Salud y comunidad. Evaluación de los recursos y estresores. Valencia: CSV.

Keyes, C. 1998. "Social well-being." Social Psychology Quarterly 61: 121-140.

Khon, M. L. 1976. "Occupational structure and alienation." American Journal of Sociology 82: 111-130.

Lazarus, R. S. y S. Folkman. 1986. Estrés y procesos cognitivos. Barcelona: Martínez Roca.

Marx, K. 1978. Manuscritos: Economía y filosofía. Madrid: Alianza.

Marx, K. 1980. El capital. Crítica de la economía política. Madrid: Siglo XXI.

McLeod, J. D. y J. M. Nonnemaker. 1999. "Social stratification and inequality." Pp. 321-344 en Handbook of the sociology of mental health, editores Aneshensel C. S. y J. C. Phelan. Nueva York: Kluwer Academia.

Mirowsky, J. y C. E. Ross. 1989. Social causes of psychological distress. Nueva York: Aldine de Gruyter.

Pearlin, L. I. 1999. "The stress process revisited: Reflections on concepts and their interrelationships." Pp. 395-416 en Handbook of the sociology of mental health, editores Aneshensel, C. S. y J. C. Phelan. Nueva York: Kluwer Academia.

Peterson, C., S. F. Maier y M. E. P. Seligman. 1993. Learned helplessness, $A$ theory for the age of personal control. Oxford: University Press.

Peterson, C., A. Semmel, C. Von Baeyer, L. Abramson, G. Metalsky y M. E. P. Seligman. 1982. "The attributional style questionnaire." Cognitive Therapy and Research 6: 287-300.

Rosenberg, M. 1965. Society and the adolescent self-image. New Jersey: Princeton University Press.

Ross, C. E. y J. Sastry. 1999. "The sense of personal control. Social-structural causes and emotional consequences." Pp. 369-394 en Handbook of the sociology of mental health, editores Aneshensel, C. S. y J. C. Phelan. Nueva York: Kluwer Academia. 
Sánchez, E., A. Garrido J. L. Álvaro. 2000. "Estrés y salud mental: El papel de la posición social y las estrategias de afrontamiento". Pp. 397-403 en Intervención psicosocial y comunitaria. La promoción de la salud y la calidad de vida, editores Fernández, J., J. Herrero y A. Bravo. Madrid: Biblioteca Nueva.

Sánchez, E., A. Garrido y J. L. Álvaro. 2003. "Un modelo psicosociológico para el estudio de la salud mental." Revista de Psicología Social 18: 17-33.

Seeman, M. 1959. "On the meaning of alienation." American Sociological Review 24: 783-791.

Seeman, M. 1983. "Alienation motifs in contemporary theorizing: The hidden continuity of classic themes." Social Psychology Quarterly 46: 171-184.

Seligman, M. E. P. 1979. "Depressive attributional style." Journal of Abnormal Psychology 88: 242-247.

Seligman, M. E. P. 1981. Indefensión. Madrid: Debate.

Southhall, D. y J. Roberts. 2002. "Attributional style and self-esteem in vulnerability to adolescence depressive symptoms following life stress. A 14-week prospective study." Cognitive Therapy and Research 26: 563-579.

Stryker, S. 1980. Symbolic Interactionism: A Social Structural Approach. Menlo Park: Benjamin/Cummings.

Sweeney, P. G., K. Anderson y S. Bailey. 1986. "Attributional style in depression: a meta-analytic review." Journal of Personality and Social Psychology 50: 974-91.

Turner, R. J. y J. B. Turner. 1999. "Social integration and support." Pp. 301-320 en Handbook of the sociology of mental health, editores Aneshensel, C. S. y J. C. Phelan. Nueva York: Kluwer Academia.

Warr, P. B. y P. R. Jackson. 1983. "Self-esteem and unemployment among young worker." Le Travail Humane 46: 355-66.

Weiner, B. 1986. "Attribution, Emotion and Action." Pp. 281-312 en Handbook of motivation and cognition, eds. Sorrentino, R. M. y E. T. Higgins. Nueva York: Guilford.

Wheaton, B. 1999. "Social stress." Pp. 277-300 en Handbook of the sociology of mental health, editores Aneshensel, C. S. y J. C. Phelan. Nueva York: Kluwer Academia.

World Health Organization. 2002. "Depresion." Geneva: World Health Organization. Consultado 28 de marzo del 2008. (http://www.who.int/mental_health/management/depression/definition/en/)

Yu, Y. y D.R. Williams. 1999. "Socioeconomic status and mental health." Pp. 151-166 en Handbook of the sociology of mental health, editores Aneshensel, C. S. y J. C. Phelan. Nueva York: Kluwer Academia. 
JOSÉ LUIS ÁLVARO ESTRAMIANA es Catedrático de Psicología Social en el Departamento de Psicología Social de la Universidad Complutense de Madrid y profesor invitado en diferentes Universidades europeas y latinoamericanas. Es autor de numerosos libros, capítulos de libros y artículos de psicología social publicados en revistas científicas nacionales e internacionales.

ALICIA GARRIDO LUQUE es Profesora Titular de Psicología Social en el Departamento de Psicología Social de la Universidad Complutense de Madrid y autora y coautora de diferentes artículos publicados en revistas nacionales e internacionales, así como libros y capítulos de libros.

INGE SCHWEIGER GALLO es profesora del Departamento de Psicología social de la Universidad Complutense de Madrid. Ha publicado artículos en revistas tanto nacionales como internacionales y sus líneas de investigación se centran en el ámbito de las emociones, las actitudes y las teorías en Psicología Social.

RECIBIDO: $08 / 06 / 08$

ACEPTADO: $15 / 04 / 09$

Publicado on-line: 22 de abril de 2010 